\title{
Chemical variations and star formation histories of damped Ly $\alpha$ systems
}

\author{
M. Dessauges-Zavadsky ${ }^{1}$, J. X. Prochaska ${ }^{2}$, S. D’Odorico $^{3}$, \\ F. Calura ${ }^{4}$, and F. Matteucci ${ }^{4,5}$ \\ ${ }^{1}$ Observatoire de Genève, $51 \mathrm{Ch}$. des Maillettes, 1290 Sauverny, Switzerland \\ ${ }^{2}$ UCO/Lick Observatory, University of California at Santa Cruz, Santa Cruz, CA 95064, USA \\ ${ }^{3}$ European Southern Observatory, Karl-Schwarzschildstr. 2, 85748 Garching, Germany \\ ${ }^{4}$ Dipartimento di Astronomia-Universitá di Trieste, Via G. B. Tiepolo 11, 34131 Trieste, Italy \\ ${ }^{5}$ INAF, Osservatorio Astronomico di Trieste, Via G. B. Tiepolo 11, 34131 Trieste, Italy
}

\begin{abstract}
Thanks to the comprehensive sets of elemental abundances in eleven damped Ly $\alpha$ systems (DLAs) at $z_{\mathrm{abs}}=1.7-2.5$, we were able for the first time to study in detail the abundance patterns of a wide range of elements, the chemical variations in the interstellar medium, the star formation, and the age of galaxies outside the Local Group. Comparing the gas-phase abundance ratios of these high redshift galaxies, we found that they are very uniform, showing low RMS dispersions up to only 2-3 times higher than the statistical errors, for the majority of elements. The gas-phase abundance patterns of interstellar medium clouds within the DLA galaxies detected along the velocity profiles show on the other hand a high dispersion in several abundance ratios, indicating that variations in the gas phase, whatever their origin, are more confined to clouds within the DLA galaxies than to integrated profiles. The analysis of the cloud-to-cloud chemical variations within seven individual DLAs revealed that five of them show statistically significant variations, higher than 0.2 dex at more than $3 \sigma$. The sources of these variations are both differential dust depletion and ionisation effects, however no evidence for variations due to different star formation histories was highlighted. These results place important constraints on scenarios of galaxy formation within the CDM hierarchical theory. The DLA abundance pattern comparison with chemical evolution models indicate that the DLAs may either be associated with the outer regions of spiral galaxies or with dwarf irregular galaxies both characterised by star formation histories with low star formation efficiencies. They may be very young galaxies with ages between $20-250 \mathrm{Myr}$, but also galaxies with ages longer than 1 Gyr. Their star formation rates per unit area are moderate, between $-3.2<\log \mathrm{SFR}<$ $-1.4 \mathrm{M}_{\odot} \mathrm{yr}^{-1} \mathrm{kpc}^{-2}$.
\end{abstract}

\section{Introduction}

In Dessauges-Zavadsky et al. (2004, hereafter DZ04), we obtained comprehensive sets of elemental abundances for four DLAs. This allowed us to study each DLA galaxy individually, while previously the DLA galaxy population has been analysed as a whole. These exciting results encouraged us to carry on the analysis of individual DLAs by extending our sample. We obtained high quality UVES/VLT spectra of seven additional DLAs. Combined with existing HIRES/Keck spectra we took column density measurements of 30 ions from 22 elements, - B, C, N, O, Mg, Al, Si, P, S, Cl, Ar, Ti, Cr, Mn, $\mathrm{Fe}, \mathrm{Co}, \mathrm{Ni}, \mathrm{Cu}, \mathrm{Zn}, \mathrm{Ge}, \mathrm{As}$, and $\mathrm{Kr}$. Altogether, we have a sample of eleven DLA galaxies with uniquely comprehensive sets of abundance measurements (see Dessauges-Zavadsky et al. 2005).

With this sample of DLAs, we investigate in section 2 the issue of chemical variations both in the abundance patterns of DLA galaxies and DLA interstellar medium (ISM) 
gas clouds along their velocity profiles, and in section 3 the issue of DLA star formation histories (SFHs) through detailed comparison with chemical evolution models. Chemical variations are expected, if the DLA galaxy population samples galaxies with different SFHs, different stages of chemical evolution, and different ISM conditions. This is suggested by low redshift deep imaging revealing a variety of morphological types associated with the DLA population (Le Brun et al. 1997; Nestor et al. 2002; Chen \& Lanzetta 2003). A range of at least 0.3 dex is observed in the $\alpha$ over iron-peak element ratios when comparing the abundance measurements of stars in the Milky Way with those of the Small and Large Magellanic Clouds (SMC, LMC) and dwarf spheroidal galaxies, and within a given galaxy, due to different SFHs (e.g. Shetrone et al. 2003). Similarly there is a large range of more than 0.5 dex in the gas-phase abundances of different lines of sight crossing the Milky Way and Magellanic Clouds due to different physical conditions, e.g. various dust-to-gas ratios, volume densities, and ionisation states within the ISM (Savage \& Sembach 1996; Welty et al. 2001).

\section{Chemical variations}

\subsection{Global gas-phase abundance patterns}

Fig. 1 shows the raw abundance patterns (free from any correction) as a function of the atomic number of the eleven DLAs from our sample. We present both the $[\mathrm{X} / \mathrm{Si}]$ and $[\mathrm{X} / \mathrm{Fe}]$ abundance ratios for the entire set of 22 detected elements $\mathrm{X}$.

The global abundance patterns of DLAs show relatively low RMS dispersions, reaching only 2-3 times higher values than the statistical errors for the majority of elements. This uniformity is remarkable given that the quasar sight-lines cross gaseous regions with H I column densities covering an order of magnitude from $2 \times 10^{20}$ to $4 \times 10^{21} \mathrm{~cm}^{-2}$ and with metallicities ranging from $1 / 55$ to $1 / 5$ Solar, and that at low redshift DLAs seem to be associated with different galaxies. This suggests that the effects of nucleosynthesis enrichment, dust depletion, and ionisation, the three possible sources of chemical variations in the gas phase, are low. In turn, this implies the respective SFHs have conspired to yield one set of relative abundances.

We discuss element per element the implications of this uniformity. The most interesting results are: (i) the $[\mathrm{Si} / \mathrm{Fe}]$ and $[\mathrm{S} / \mathrm{Fe}]$ abundance ratios show an $\alpha$-enhancement irrespective of the dust depletion level of the DLAs studied; and (ii) to reconcile this $\alpha$-enhancement with the Solar $[\mathrm{Si} / \mathrm{Zn}]$ ratios, we suggest that the $[\mathrm{Zn} / \mathrm{Fe}]$ ratios are overSolar independently from dust depletion effects, as indicated by the recent measurements in Galactic metal-poor stars (e.g. Nissen et al. 2004).

\subsection{Gas-phase abundance patterns of individual clouds}

From the Voigt profile fitting of metal-lines, we obtained very accurate component-tocomponent column density measurements. These components presumably correspond to interstellar medium "clouds" in the DLA galaxy on the quasar line of sight.

By considering together all the clouds of all the DLAs studied (84 clouds), we notice that in general the mean relative abundances of clouds are very similar to those of the integrated profiles of DLAs, but the measured RMS dispersions and reduced $\chi^{2}$ for the clouds are $2-3$ times larger. Indeed, we observe a statistically significant dispersion in several abundance ratios. This indicates that the chemical variations are more confined to individual clouds within the DLA galaxies rather than to the integrated profiles.

We found unambiguous correlations between [Si/Fe], [S/Fe], and $[\mathrm{S} / \mathrm{Si}]$ versus $[\mathrm{Zn} / \mathrm{Fe}]$; and anti-correlations between $[\mathrm{Si} / \mathrm{Zn}]$ and $[\mathrm{S} / \mathrm{Zn}]$ versus $[\mathrm{Zn} / \mathrm{Fe}]$ (Fig. 2). These trends are primarily the result of differential dust depletion effects, which is also responsible for the 

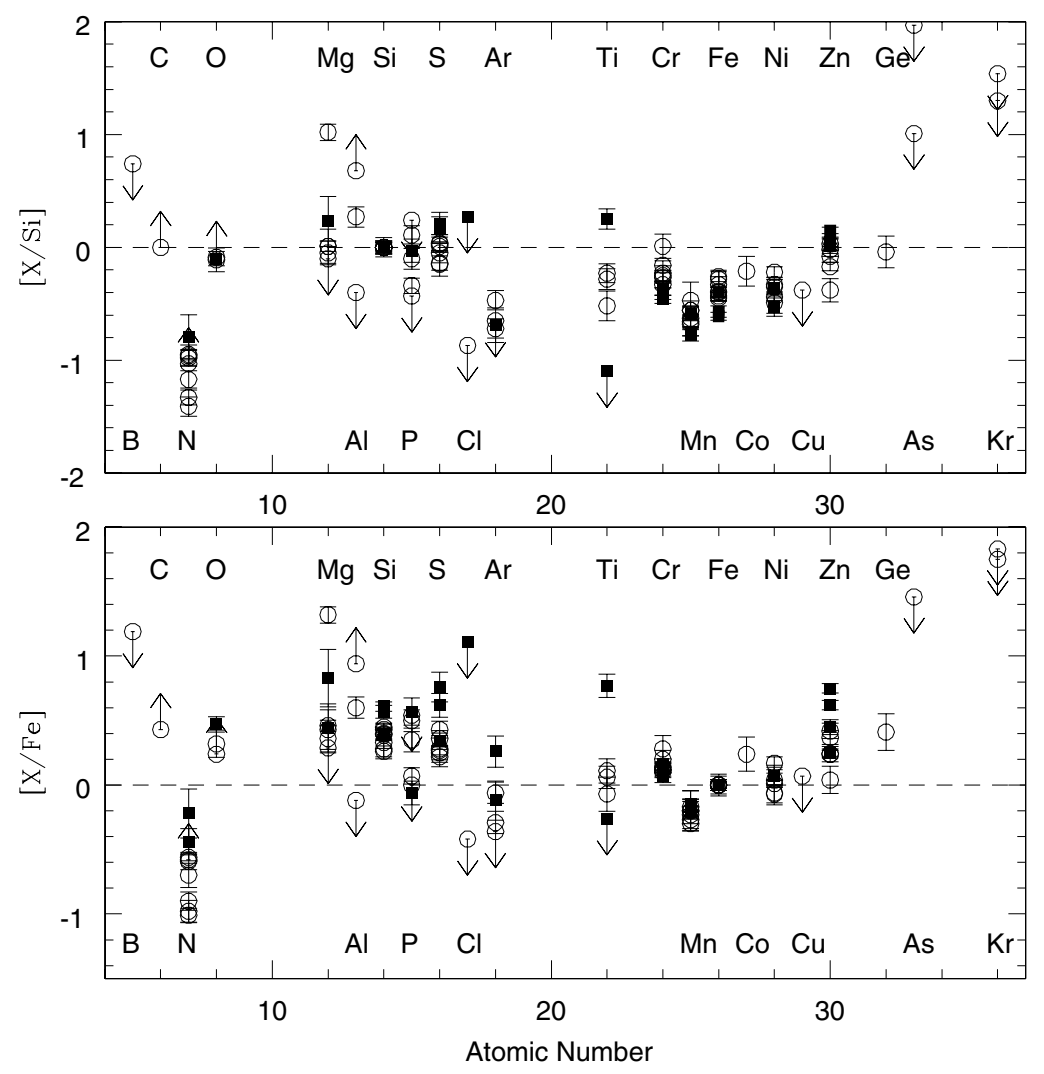

Figure 1. Abundance patterns [X/Si] (upper panel) and $[\mathrm{X} / \mathrm{Fe}]$ (lower panel) for our sample of 11 DLAs. The plotted abundance ratios are the raw abundance ratio measurements, i.e. free from any correction. Our sample of data is composed of the 7 DLAs studied in Dessauges-Zavadsky, Prochaska, D'Odorico et al. (2005) (circles) and of 4 DLAs analysed in DZ04 (squares).

high cloud-to-cloud abundance ratio dispersions. The signature of a pure nucleosynthesis enrichment contribution can be observed in the $[\alpha / \mathrm{Fe}, \mathrm{Zn}]$ ratios at low dust depletion levels, $[\mathrm{Zn} / \mathrm{Fe}]<0.2$. It is characterised by an $\alpha$-enhancement in the clouds. However, while the $[\mathrm{Si} / \mathrm{Fe}],[\mathrm{S} / \mathrm{Fe}]$, and $[\mathrm{Si} / \mathrm{Zn}]$ ratios are highly $\alpha$-enhanced $(>0.2 \mathrm{dex}),[\mathrm{S} / \mathrm{Zn}]$ remains almost Solar $(+0.13 \pm 0.08)$, suggesting that $[\mathrm{S} / \mathrm{Zn}]$ may not be a reliable tracer of nucleosynthesis enrichment.

\subsection{Cloud-to-cloud chemical variations in individual DLAs}

There are three possible sources of abundance variations from cloud-to-cloud: (i) different nucleosynthesis enrichments; (ii) different dust depletion levels; and (iii) different ionisation conditions. Analysing specific abundance ratios, we tried to determine the sources responsible for chemical variations when observed in a given DLA galaxy.

The study of the cloud-to-cloud chemical variations within seven individual DLAs revealed that five of them show statistically significant variations, higher than 0.2 dex at more than $3 \sigma$. Two of them show "extreme" variations with a dispersion higher than 0.3 dex at more than $7 \sigma$. The sources of these variations are either differential dust depletion or ionisation effects. There is no evidence for variations due to different SFHs. This suggests that the gas clouds within DLA galaxies have different physical properties, but they all seem to show a uniform nucleosynthetic enrichment history. 

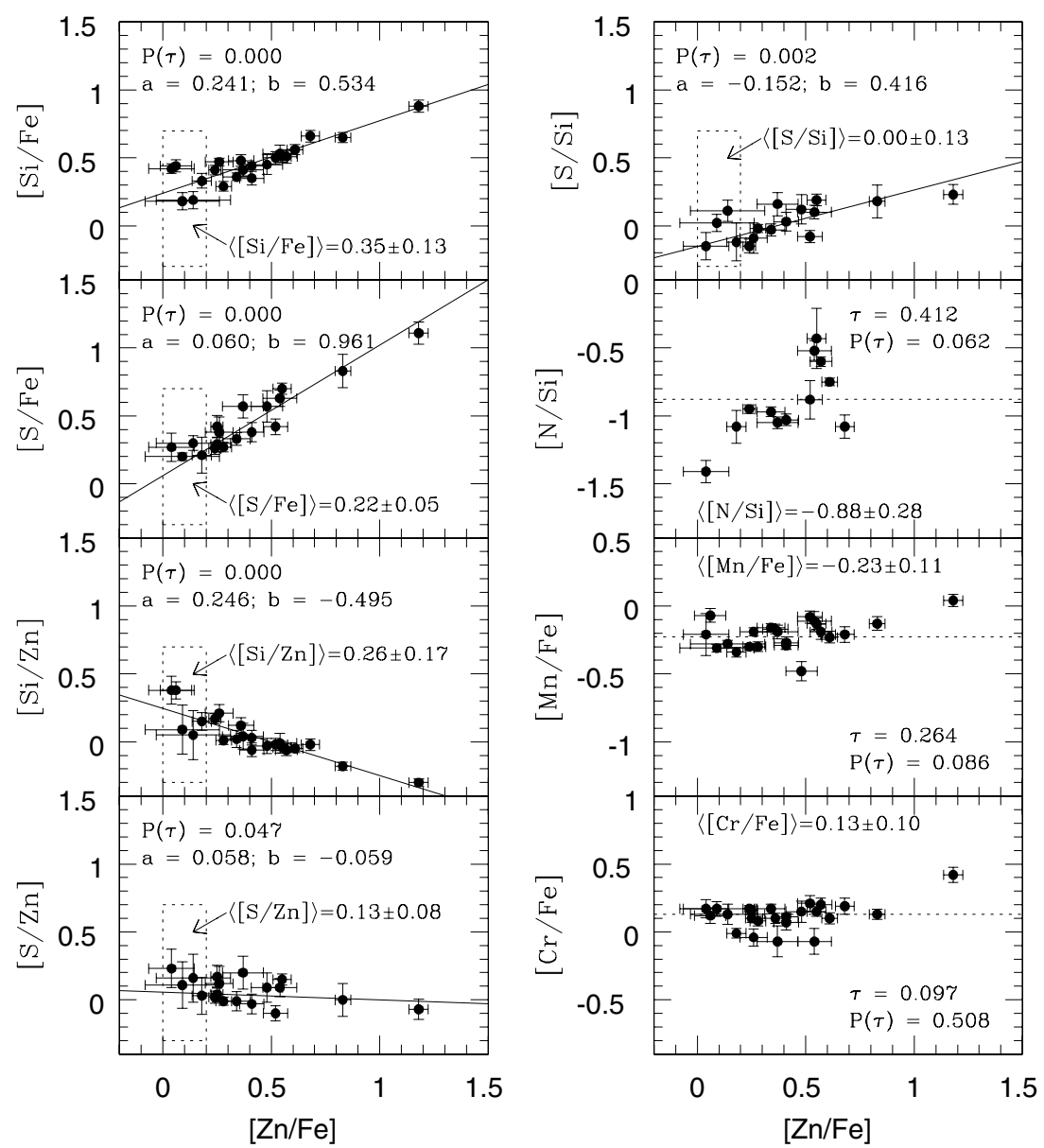

Figure 2. $[\mathrm{X} / \mathrm{Y}]$ versus $[\mathrm{Zn} / \mathrm{Fe}]$ for the entire set of clouds observed in our sample of 11 DLAs. The Kendall test shows correlations between $[\mathrm{Si} / \mathrm{Fe}],[\mathrm{S} / \mathrm{Fe}],[\mathrm{Si} / \mathrm{Zn}],[\mathrm{S} / \mathrm{Zn}]$, [S/Si], and $[\mathrm{Zn} / \mathrm{Fe}]$ with a probability under the null hypothesis of zero correlation $\mathrm{P}(\tau)<5 \%$. The derived linear least-square regressions, $[\mathrm{X} / \mathrm{Y}]=\mathrm{a}+\mathrm{b} \times[\mathrm{Zn} / \mathrm{Fe}]$, computed by taking into account the errors on both $[\mathrm{Zn} / \mathrm{Fe}]$ and $[\mathrm{X} / \mathrm{Y}]$ data points, are the solid lines. In panels $1-5$ we note the weighted mean of $[\mathrm{X} / \mathrm{Y}]$ in the interval $0<[\mathrm{Zn} / \mathrm{Fe}]<0.2$ (data points contained in the dotted boxes).

The uniformity in the nucleosynthesis enrichment observed within all DLAs is surprising and poses important constraints on the nature of high-redshift galaxies. Indeed, one very promising scenario which allows us to explain the DLA kinematics is within the CDM hierarchical cosmology and describes a DLA as multiple merging "clumps" bound to individual dark matter halos (Haehnelt, Steinmetz \& Rauch 1998; Maller et al. 2001). In this scenario, the uniformity of cloud-to-cloud abundance ratios within a DLA galaxy constrains the abundances of all the protogalactic clumps making up a DLA system. In terms of nucleosynthesis enrichment, this implies the clumps share a similar chemical enrichment pattern. This represents a challenge for the CDM simulations, since these protogalactic clumps, which do not share a common gas reservoir and which merge over a large timescale, may have a unique enrichment history and the same stage of chemical evolution. It remains to be demonstrated whether these clumps might really express very similar nucleosynthetic enrichment patterns with, in addition, 
in some cases very different dust depletions. This places also strict constraints on the mixing timescales of protogalaxies. The Access to the metallicities of these clouds is needed to confirm these statements. However, for this we need to get the H I column density of individual clouds along a quasar line of sight, which is at the present time not possible.

\section{Star formation histories}

Theoretically we can determine the SFH and the age of a galaxy from its chemical abundance measurements. Indeed, the relative abundances versus the absolute abundances constrain the SFH, while the relative abundances versus the redshift constrain the age (Matteucci 2001). However, to carry out this exercise we need to know the intrinsic abundances. These comprehensive sets of ions and elemental abundances allowed us to constrain both the dust depletion and ionisation effects in eight DLAs from our sample.

\subsection{Chemical evolution models}

A chemical evolution model allows us to follow in detail the evolution of the abundances of several chemical species, starting from the matter reprocessed by stars and restored into the ISM through stellar winds and supernova explosions. We consider in this work the grids of two models, which we identify as the "spiral model" and the "dwarf irregular model" according to the type of galaxies they match best. These models are the same as those used by Calura, Matteucci \& Vladilo (2003).

The spiral model is calibrated on the chemical features of the Milky Way (Chiappini, Matteucci \& Gratton 1997; Chiappini, Matteucci \& Romano 2001). We consider different galactocentric radii $R$ from 2 to $18 \mathrm{kpc}$. The dwarf irregular model prescriptions can be found in Bradamante, Matteucci \& D'Ercole (1998). In this model star formation (SF) can proceed either in SF bursts separated by quiescent periods, or in a low-level SF regime but continuously. Three free parameters can be adjusted in this model, the burst SF efficiency $\nu$, the burst duration $\Delta t$, and the time of occurrence of the burst $t_{b}$.

\subsection{DLA comparison with chemical evolution models}

The abundance ratios that help to constrain the SFH and the age of the DLA galaxy by means of a detailed comparison with a grid of spiral and dwarf irregular models are [Si/Fe], [S/Fe], [S/Zn], [O/Zn], [Mg/Fe], [Ni/Fe], [N/Si], [N/S], and [N/O]. This work shows that the DLAs may either be associated with the outer regions of spiral galaxies $(R>8 \mathrm{kpc})$ or with dwarf irregular galaxies both showing SFHs characterised by low SF efficiencies. Observed at $z_{\text {abs }}=1.7-2.5$, they may be very young galaxies with ages between $50-250$ Myr likely experiencing their first SF episodes, but also galaxies with ages longer than 1 Gyr (left panel of Fig. 3). From the model comparison we have, in addition, a direct access to the star formation rates (SFRs) of these DLA galaxies. The derived star formation rates per unit area are moderate, between $-3.2<\log \mathrm{SFR}<$ $-1.4 \mathrm{M}_{\odot} \mathrm{yr}^{-1} \mathrm{kpc}^{-2}$. They are in good agreement with the interval of SFR values obtained by Wolfe, Prochaska \& Gawiser (2003) and are similar to the SFR measured in the Milky Way ISM, but are much lower than the SFRs derived for the star forming galaxies at the same redshifts (Savaglio et al. 2004; right panel of Fig. 3).

\section{Acknowledgements}

M. D.-Z. was supported by Swiss National Funds and thanks the UCO/Lick Observatory for hosting her in September 2004, where part of these results were worked out. 

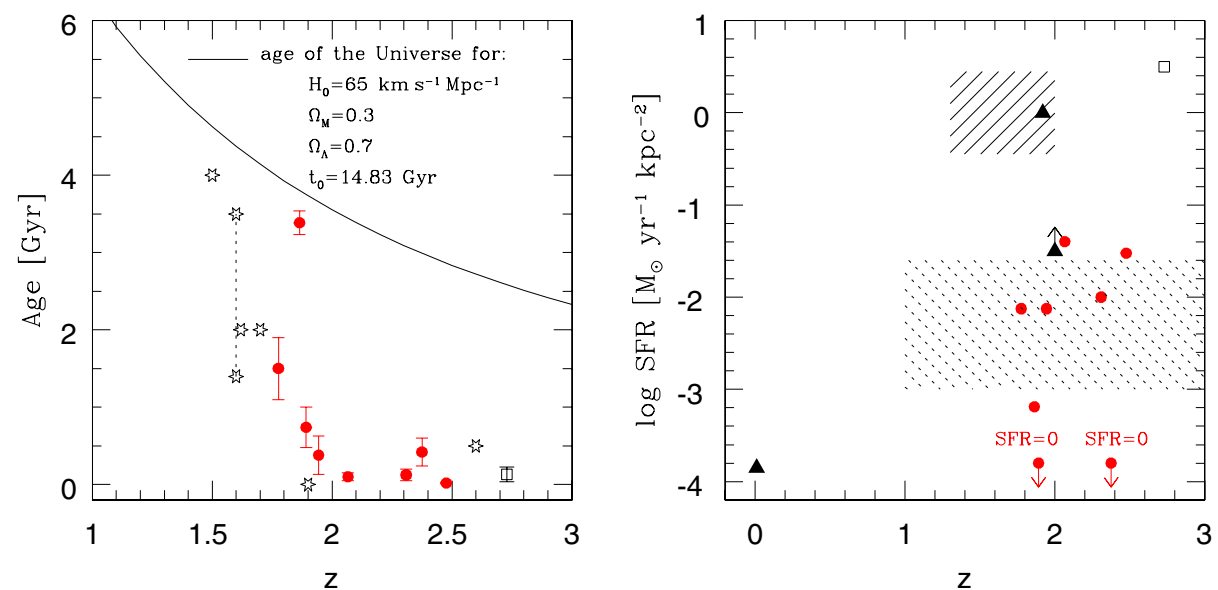

Figure 3. Left panel. Age versus redshift for the DLA galaxies studied (circles) and for galaxies analysed via integrated spectra (stars, Bruzual 2002). Right panel. SFR versus redshift. Our DLA results are shown by circles. The dotted area represents the SFRs obtained for the DLAs by Wolfe, Prochaska \& Gawiser (2003), and the dashed area the SFRs obtained for the star forming galaxies from GDDS (Savaglio et al. 2004). The triangles correspond to two DLAs for which we have an estimation of the SFR from emission lines, and the square corresponds to the Lyman-break galaxy MS 1512-cB58 (Pettini et al. 2000).

\section{References}

Bradamante, F., Matteucci, F., D'Ercole, A., 1998, A\&A, 337, 338

Bruzual, G. A., 2002, Revista Mexicana de Fisica, astro-ph/0202178

Calura, F., Matteucci, F., Vladilo, G., 2003, MNRAS, 340, 59

Chen, H.-W., Lanzetta, K. M., 2003, ApJ, 597, 706

Chiappini, C., Matteucci, F., Gratton, R., 1997, ApJ, 477, 765

Chiappini, C., Matteucci, F., Romano, D., 2001, ApJ, 554, 1044

Dessauges-Zavadsky, M., Calura, F., Prochaska, J. X., D’Odorico, S., Matteucci, F., 2004, A\&A, 416, 79 [DZ04]

Dessauges-Zavadsky, M., Prochaska, J. X., D’Odorico, S., Calura, F., Matteucci, F., 2005, A\&A, submitted

Haehnelt, M. G., Steinmetz, M., Rauch, M., 1998, ApJ, 495, 647

Le Brun, V., Bergeron, J., Boissé, P., Deharveng, J. M., 1997, A\&A, 321, 733

Maller, A. H., Prochaska, J. X., Somerville, R. S., Primack, J. R., 2001, MNRAS, 326, 1475

Matteucci, F., 2001, Dordrecht: Kluwer Academic Publisher, The Chemical Evolution of the Galaxy, Astrophysics and Space Science Library, vol. 253

Nestor, D. B., Rao, S. M., Turnshek, D. A., Monier, E., Lane, W. M., Bergeron, J., 2002, in: J. S. Mulchaey \& J. Stocke (eds.), Extragalactic Gas at Low Redshift, APS Conf. Ser., vol. 254 , p. 34

Nissen, P. E., Chen, Y. Q., Asplund, M., Pettini, M., 2004, A\&A, 415, 993

Pettini, M., Steidel, C. C., Adelberger, K. L., Dickinson, M., Giavalisco, M., 2000, ApJ, 528, 96

Savage, B. D., Sembach, K. R., 1996, ARA\&A, 34, 279

Savaglio, S., Glazebrook, K., Abraham, R. G., Crampton, D., Chen, H.-W., McCarthy, P. J. P., Jorgensen, I., et al., 2004, ApJ, 602, 51

Shetrone, M., Venn, K. A., Tolstoy, E., Primas, F., Hill, V., Kaufer, A., 2003, AJ, 125, 684

Welty, D. E., Lauroesch, J. T., Blades, J. C., Hobbs, L. M., York, D. G., 2001, ApJ, 554, 75

Wolfe, A. M., Prochaska, J. X., Gawiser, E., 2003, ApJ, 593, 215 